\section{Establishing the diagnosis neurofibromatosis type 1: A rare case}

\author{
Dyatiara Devy, Damayanti \\ Departement of Dermatology and \\ Venereology, Faculty of Medicine, \\ Universitas Airlangga / Dr.Soetomo \\ General Hospital, Surabaya, Indonesia
}

\begin{abstract}
Neurofibromatosis type 1 (NF1) or Von Recklinghausen's disease is a dominantly inherited genetic, multisystem disorder. Individuals with neurofibromatosis type 1 are prone to develop benign and malignant tumors of the CNS and peripheral nervous system, in addition to malignant diseases affecting other parts of the body. About $50 \%$ of individuals with neurofibromatosis type 1 have no family history of the disease and the disease is due to de novo (spontaneous) mutations. Early diagnosis is challenging because of its extremely variable characteristics. Some individuals may be mildly affected showing minimal signs, whereas others are severely afflicted. Individuals with NF-1 are best cared for within a multidisciplinary clinic, which has access to a wide range of subspecialists. The dermatologist has a role not only in the diagnosis of NF1 and differentiating it from other similar disorders, but also in the recognition of rare associated skin manifestations.
\end{abstract}

\section{Introduction}

Neurofibromatosis type 1 (NF1) or Von Recklinghausen's disease is a rare genetic disorder that affects about one in 2500 to one in 3000 people world wide. Neurofibromatosis type 1 is a dominantly inherited genetic disorder that results from a germline mutation in the NF1 tumoursuppressor gene. NF1 is located on chromosome 17q11.2 and encodes a 220 $\mathrm{kDa}$ cytoplasmic protein called neurofibromin. This protein functions, in part, as a negative regulator of the Ras proto-oncogene, which is a key signalling molecule in the control of cell growth. About $50 \%$ of individuals with neurofibromatosis type 1 have no family history of the disease and the disease is due to de novo (spontaneous) mutations. ${ }^{1}$ Variable characteristic showing in individual of NF1. Some individuals may be mildly affected showing minimal signs, whereas others are severely afflicted. ${ }^{2}$ NF1 is diagnosed through clinical assessment including a thorough history and physical examination using National Institute of Health Consensus Development Conference criteria. ${ }^{2,3}$ The seven diagnostic features recognized at this conference, and the recommendation that two or more of these seven features be present before a diagnosis of NF-1 is stablished. ${ }^{4}$ Neurofibromatosis type 1 manifest as not only cutaneous but also affect nearly every organ system. Dermatologists should quickly recognize not only the salient skin features but also the less common cutaneous findings, as these latter aspects may be the source of a referral. ${ }^{5}$ The most frequent features of NF1 include neurofibromas and café au lait spots. About $95 \%$ individuals with NF-1 shows café au lait spots, and $50 \%$ have plexiform neurofibromas. ${ }^{2}$ Despite the identification of the NF-1 gene and its complete sequencing, the diagnosis of NF-1 remains primarily a clinical one. ${ }^{4}$

\section{Case Report}

A 5-year-old girl presented with multiple hyperpigmented macule since birth. The macule became wider and multiplied in growth. No history with the same disease in her family (Figure 1). From physical examination, there were multiple café-au-lait spots with diameter more than $0.5 \mathrm{~cm}$ all over the body, plexiform neurofibroma, and axillary freckling (Figure 2). At this moment, this patient was diagnosed with neurofibromatosis type 1 . We consult this patient to ophtalmologist, pediatricians and do the routine blood examination. The standard laboratory tests values were in the normal range. Lisch's nodules on the iris of both eyes were found without clinical visual involvement, and from the CT Scan result showed no tumor in optic pathway. The pediatricians did not detect alterations in the central and peripheral nervous system.

\section{Discussion}

In childhood or early adolescence, the characteristic clinical features neurofibromatosis type 1 would be apparent. The diagnosis NF-1 was made according to the presence of two or more diagnostic criteria of the National Institute of Health Consensus Development Conference (Table 1). ${ }^{1-7}$ In this case, we found 4 point indicates as neurofibromatosis. The café-au-lait macule with more than $0,5 \mathrm{~cm}$ in diameter is one of the seven cardinal diagnostic criteria of NF1 was found in this case more than six.
Correspondence: Dyatiara Devy, Department of Dermatology and Venerology, Faculty of Medicine, Universitas Airlangga/ Dr. Soetomo General Hospital, J1. Prof. Dr. Moestopo no. 47, Surabaya, Indonesia.

Telephone/fax: 081216390735

E-mail: devy_doctor@yahoo.com

Key words: Neurofibromatosis type 1, Diagnosis, Multidisciplinary.

Contributions: All the authors contributed equally.

Conflict of interests: The authors declare no potential conflict of interest.

Received for publication: 1 February 2019. Accepted for publication: 25 February 2019.

This work is licensed under a Creative Commons Attribution-NonCommercial 4.0 International License (CC BY-NC 4.0).

(C) Copyright D. Devy and Damayanti, 2019 Licensee PAGEPress, Italy

Dermatology Reports 2019; 11(s1):8092 doi:10.4081/dr.2019.8092

Café-au-lait spots, which are flat, pigmented macules, are often the first manifestation of NF-1 to appear. Frequently present at birth, they become more numerous as the infant grows. ${ }^{4}$ About 95$99 \%$ individu with neurofibromatosis shows café-au-lait spots. ${ }^{2,5}$ Axillary and inguinal freckling is another common clinical feature of neurofibromatosis type 1 and is usually detected in affected individuals by age 5-8 years. These pigmentary abnormalities are typically the second diagnostic characteristic seen in children with the disorder, generally arising after development of cafe au- lait macules.

Plexiform neurofibromas develop in about $30-50 \%$ of individuals with neurofibromatosis type 1, typically manifest at birth but can continue to grow during adolescence and early adulthood, plexiform neurofibromas may manifest as diffuse appearance and/or a tendency to expand along large segments of affected nerves, causing disfigurement and nerve dysfunction. ${ }^{7}$ In this case there were plexiform neurofibromas with no disfigurement of the other structure. Yearly eye examinations by an ophthalmologist are essential in children under the age of 10 and may be necessary in older children. It is important for the health care provider to detect any visual changes. The common finding of ophthalmic abnormalities is lisch nodules, which are small, domeshaped hyperpigmented macules of the iris that cause no impairment of vision. They are a 


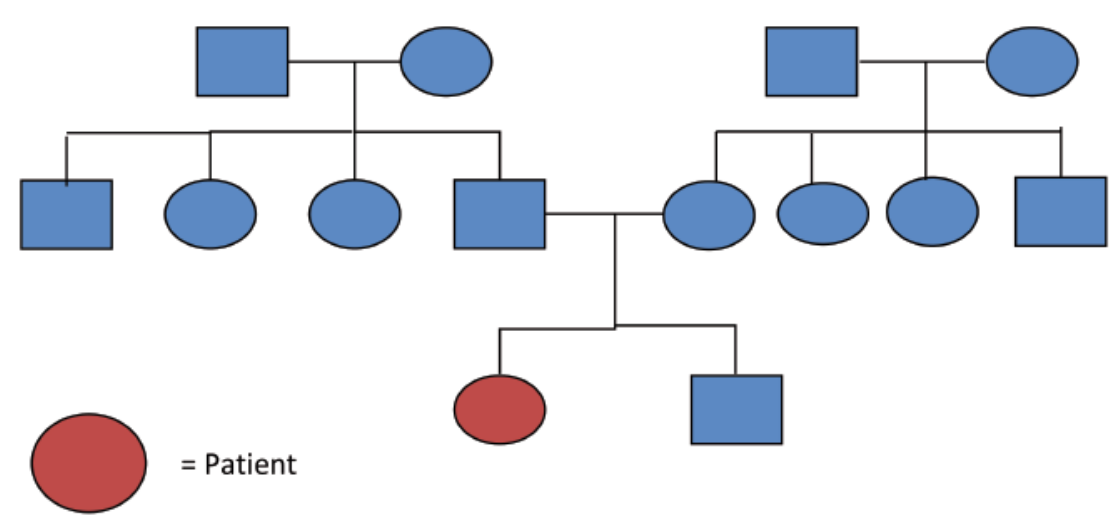

Figure 1. Patient's pedigree.

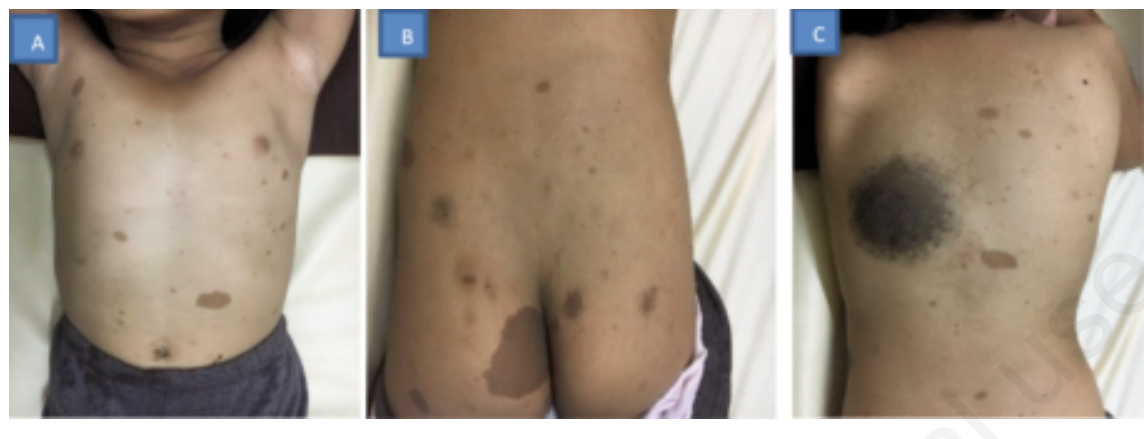

Figure 2. (A) Axillary freckling, (B) Café-au-lait spots with variation in diameter, (C) Plexiform neurofibromas.

Table 1. Diagnostic criteria of the National Institute of Health Consensus Development Conference. ${ }^{1-7}$

\section{Clinical diagnostic criteria for neurofibromatosis-1 (NF-1)}

Patients have two or more of the following symptoms :

a) 6 or more café-au-lait macules ( $>0.5 \mathrm{~cm}$ in children of $>1.5 \mathrm{~cm}$ in adults)

b) 2 or more cutaneous or subcutaneous neurofibromas or one plexiform neurofibroma

c) Axillary or groin freckling

d) Optic glioma

e) 2 or more Lisch nodules (iris hamartomas visualized on slit lamp examination)

f) Sphenoid wing dysplasia or bowing of long bone (with or without pseudarthrosis)

g) First degree relative with an NF1 diagnosis common finding by more than 3 years of age of children and are included as one of the cardinal NIH diagnostic criteria..$^{2,6}$

\section{Conclusions}

Neurofibromatosis type 1 is a multisystem disorder requiring management by multiple disciplines. However, the diagnosis is missed on many individuals. The dermatologist has a role to play in the diagnosis of NF1, differentiating it from other similar disorders, and also recognition of rare but associated skin manifestations.

\section{References}

1. Hirbe AC, Gutmann DH Neurofibromatosis type 1: a multidisciplinary approach to care, Lancet Neurol 2014;13:834-43.

2. Julian N, Edwards NE, DeCrane S, Hingtgen CM. Neurofibromatosis 1: diagnosis and management. JNP 2014;10:30-5

3. Sayah C, Benmahmoud M, Yahia SA, Soualili Z. Neurofibromatosis type 1 (NF1): case report and review of literature. J Child Dev Disord 2016;2:1-4.

4. Listernick R, Charrow J. The neurofibromatoses. In: Wolff K, Goldsmith LA, Katz SI, Gilchrest BA, Paller AS, Leffel DJ, editors. Fitzpatrick's Dermatology in General Medicine. 8th ed. New York: McGraw Hill Companies; 2012. pp. 1680-90.

5. Ferner RE, Huson SM, Thomas N, et al. Guidelines for the diagnosis and management of individuals with neurofibromatosis. J Med Genet 2007;44:81-8.

6. Boyd KP, Korf BR, Theos A. Neurofibromatosis type 1. J Am Acad Dermatol 2009;61:1-16.

7. Gerber PA, Antal AS, Neumann NJ, et al. Neurofibromatosis. Eur J Med Res 2009;14:102-5. 